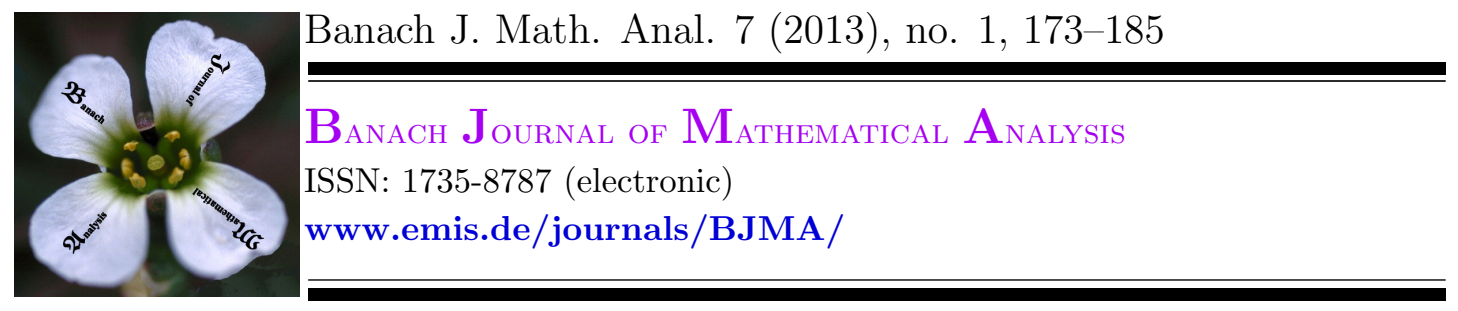

\title{
A FUBINI THEOREM ON A FUNCTION SPACE AND ITS APPLICATIONS
}

\author{
HYUN SOO CHUNG, JAE GIL CHOI AND SEUNG JUN CHANG* \\ Communicated by M. Skeide
}

\begin{abstract}
In this paper we establish a Fubini theorem for functionals on a function space. We then establish some relationships as applications of our Fubini theorem. Finally, we present some historical remarks.
\end{abstract}

\section{INTRODUCTION}

Let $C_{0}[0, T]$ denote one-parameter Wiener space; that is the space of real-valued continuous functions $x$ on $[0, T]$ with $x(0)=0$. In [1], Bearman established a very useful theorem which is called the rotation theorem on Wiener space $C_{0}[0, T]$. In $[13,14,15]$, Huffman, Park and Skoug used the rotation theorem to obtain a basic relationship between the analytic Fourier-Feynman transform and the convolution product of functionals on $C_{0}[0, T]$, and in $[16,17]$, Huffman, Skoug and Storvick established a Fubini theorem via the rotation theorem to obtain various analytic Wiener and Feynman integrals and integration formulas involving Fourier-Feynman transforms on $C_{0}[0, T]$.

In [3], Cameron and Storvick established a very fundamental result to evaluate the analytic Feynman integral for unbounded functionals on $C_{0}[0, T]$. In [20], Park, Skoug and Storvick used the fundamental result to obtain integration by parts formulas for analytic Feynman integrals and for analytic Fourier-Feynman transforms.

Date: Received: 3 April 2012; Revised: 16 August 2012; Accepted: 10 September 2012.

* Corresponding author.

2010 Mathematics Subject Classification. Primary 46G12; Secondary 47A60, 28 C20.

Key words and phrases. Generalized Brownian motion process, Fubini theorem, CameronStorvick type theorem, translation theorem, first variation. 
The function space $C_{a, b}[0, T]$, induced by generalized Brownian motion, was introduced by Yeh in [22] and was used extensively in [4, 5, 6, 7, 8, 9].

In this paper, we establish a Fubini theorem for functionals on the function space $C_{a, b}[0, T]$. We then use the Fubini theorem and the translation theorem to obtain a Cameron-Storvick type theorem. Finally, we present some historical remarks for our main results.

The Wiener process used in $[1,2,3,10,11,12,13,14,15,16,17,20]$ is free of drift and is stationary in time while the stochastic process used in this paper, as well as in $[4,5,6,7,8,9]$, is nonstationary in time, is subject to a drift $a(t)$, and can be used to explain the position of the Ornstein-Uhlenbeck process in an external force field [19]. Thus the formulas and results in this paper are more complicated than the formulas and results in [1,3]. However, when $a(t) \equiv 0$ and $b(t)=t$ on $[0, T]$, the function space $C_{a, b}[0, T]$ reduces to the Wiener space $C_{0}[0, T]$ and so the results in $[1,3,13,14,15,16,17,20]$ follow immediately from the results in this paper.

\section{Preliminaries}

Let $D=[0, T]$ and let $(\Omega, \mathcal{B}, P)$ be a probability measure space. A real-valued stochastic process $Y$ on $(\Omega, \mathcal{B}, P)$ and $D$ is called a generalized Brownian motion process if $Y(0, \omega)=0$ almost everywhere and for $0=t_{0}<t_{1}<\cdots<t_{n} \leq T$, the $n$-dimensional random vector $\left(Y\left(t_{1}, \omega\right), \ldots, Y\left(t_{n}, \omega\right)\right)$ is normally distributed with density function

$$
\begin{aligned}
W_{n}(\vec{t}, \vec{\eta}) & =\left((2 \pi)^{n} \prod_{j=1}^{n}\left(b\left(t_{j}\right)-b\left(t_{j-1}\right)\right)\right)^{-1 / 2} \\
& \cdot \exp \left\{-\frac{1}{2} \sum_{j=1}^{n} \frac{\left(\left(\eta_{j}-a\left(t_{j}\right)\right)-\left(\eta_{j-1}-a\left(t_{j-1}\right)\right)\right)^{2}}{b\left(t_{j}\right)-b\left(t_{j-1}\right)}\right\}
\end{aligned}
$$

where $\vec{\eta}=\left(\eta_{1}, \ldots, \eta_{n}\right), \eta_{0}=0, \vec{t}=\left(t_{1}, \ldots, t_{n}\right), a(t)$ is an absolutely continuous real-valued function on $[0, T]$ with $a(0)=0, a^{\prime}(t) \in L^{2}[0, T]$, and $b(t)$ is a strictly increasing, continuously differentiable real-valued function with $b(0)=0$ and $b^{\prime}(t)>0$ for each $t \in[0, T]$.

As explained in [21, pp.18-20], $Y$ induces a probability measure $\mu$ on the measurable space $\left(\mathbb{R}^{D}, \mathcal{B}^{D}\right)$ where $\mathbb{R}^{D}$ is the space of all real-valued functions $x(t), t \in D$, and $\mathcal{B}^{D}$ is the smallest $\sigma$-algebra of subsets of $\mathbb{R}^{D}$ with respect to which all the coordinate evaluation maps $e_{t}(x)=x(t)$ defined on $\mathbb{R}^{D}$ are measurable. The triple $\left(\mathbb{R}^{D}, \mathcal{B}^{D}, \mu\right)$ is a probability measure space. This measure space is called the function space induced by the generalized Brownian motion process $Y$ determined by $a(\cdot)$ and $b(\cdot)$.

We note that the generalized Brownian motion process $Y$ determined by $a(\cdot)$ and $b(\cdot)$ is a Gaussian process with mean function $a(t)$ and covariance function $r(s, t)=\min \{b(s), b(t)\}$. By Theorem 14.2 [21, p.187], the probability measure $\mu$ induced by $Y$, taking a separable version, is supported by $C_{a, b}[0, T]$ (which is equivalent to the Banach space of continuous functions $x$ on $[0, T]$ with $x(0)=0$ under the sup norm). Hence $\left(C_{a, b}[0, T], \mathcal{W}\left(C_{a, b}[0, T]\right), \mu\right)$ is the function space 
induced by $Y$ where $\mathcal{W}\left(C_{a, b}[0, T]\right)$ is the collection of all Wiener measurable subsets of $C_{a, b}[0, T]$.

A subset $E$ of $C_{a, b}[0, T]$ is said to be scale-invariant measurable provided $\rho E \in$ $\mathcal{W}\left(C_{a, b}[0, T]\right)$ for all $\rho>0$, and a scale-invariant measurable set $N$ is said to be a scale-invariant null set provided $\mu(\rho N)=0$ for all $\rho>0$. A property that holds except on a scale-invariant null set is said to hold scale-invariant almost everywhere.

Let $L_{a, b}^{2}[0, T]$ be the Hilbert space of functions on $[0, T]$ which are Lebesgue measurable and square integrable with respect to the Lebesgue Stieltjes measures on $[0, T]$ induced by $a(\cdot)$ and $b(\cdot)$; i.e.,

$$
L_{a, b}^{2}[0, T]=\left\{v: \int_{0}^{T} v^{2}(s) d b(s)<\infty \text { and } \int_{0}^{T} v^{2}(s) d|a|(s)<\infty\right\}
$$

where $|a|(t)$ denotes the total variation of the function $a(\cdot)$ on the interval $[0, t]$.

For $u, v \in L_{a, b}^{2}[0, T]$, let

$$
(u, v)_{a, b}=\int_{0}^{T} u(t) v(t) d[b(t)+|a|(t)]
$$

Then $(\cdot, \cdot)_{a, b}$ is an inner product on $L_{a, b}^{2}[0, T]$ and $\|u\|_{a, b}=\sqrt{(u, u)_{a, b}}$ is a norm on $L_{a, b}^{2}[0, T]$. In particular note that $\|u\|_{a, b}=0$ if and only if $u(t)=0$ a.e. on $[0, T]$. Furthermore $\left(L_{a, b}^{2}[0, T],\|\cdot\|_{a, b}\right)$ is a separable Hilbert space. Note that all functions of bounded variation on $[0, T]$ are elements of $L_{a, b}^{2}[0, T]$. Also note that if $a(t) \equiv 0$ and $b(t)=t$, then $L_{a, b}^{2}[0, T]=L^{2}[0, T]$. In fact,

$$
\left(L_{a, b}^{2}[0, T],\|\cdot\|_{a, b}\right) \subset\left(L_{0, b}^{2}[0, T],\|\cdot\|_{0, b}\right)=\left(L^{2}[0, T],\|\cdot\|_{2}\right)
$$

since the two norms $\|\cdot\|_{0, b}$ and $\|\cdot\|_{2}$ are equivalent. For $v \in L_{a, b}^{2}[0, T]$, let

$$
\left(v, a^{\prime}\right)=\int_{0}^{T} v(t) a^{\prime}(t) d t=\int_{0}^{T} v(t) d a(t)
$$

and

$$
\left(v^{2}, b^{\prime}\right)=\int_{0}^{T} v^{2}(t) b^{\prime}(t) d t=\int_{0}^{T} v^{2}(t) d b(t)
$$

It is well-known that for each $v \in L_{a, b}^{2}[0, T]$, the Paley-Wiener-Zygmund(PWZ) stochastic integral $\langle v, x\rangle$, see $[4,5,7,8,9]$, exists for $\mu$-a.e. $x \in C_{a, b}[0, T]$.

Throughout this paper we will assume that each functional $F: C_{a, b}[0, T] \rightarrow \mathbb{C}$ consider is scale-invariant measurable and that

$$
\int_{C_{a, b}[0, T]}|F(\rho x)| d \mu(x)<\infty
$$

for each $\rho>0$.

Remark 2.1. Let $w(t)=\frac{a^{\prime}(t)}{b^{\prime}(t)}$. In addition to the conditions put on $a(t)$ above we now add the condition

$$
\int_{0}^{T}\left|a^{\prime}(t)\right|^{2} d|a|(t)<\infty
$$


from which it follows that

$$
\int_{0}^{T} w^{2}(t) d[b(t)+|a|(t)]=\int_{0}^{T}\left[\frac{a^{\prime}(t)}{b^{\prime}(t)}\right]^{2} d[b(t)+|a|(t)]<\infty .
$$

Note that $a(t)=\int_{0}^{t} w(s) d b(s)$ for $t \in[0, T]$.

\section{A Fubini THEOREM}

In this section we define two transforms on function spaces. We then use these transforms and the translation theorem on $C_{a, b}[0, T]$ to obtain a Fubini theorem, see Theorem 4.2 below.

First, we define two interesting transforms $R_{\theta}$ and $A_{\theta}$ on $C_{a, b}^{2}[0, T] \equiv C_{a, b}[0, T] \times$ $C_{a, b}[0, T]$ used in this paper.

For each real number $\theta$, let $R_{\theta}: C_{a, b}^{2}[0, T] \rightarrow C_{a, b}^{2}[0, T]$ be the transform defined by $R_{\theta}(x, y)=(X, Y)$ where

$$
\left(\begin{array}{l}
X(t) \\
Y(t)
\end{array}\right)=\left(\begin{array}{cc}
\cos \theta & -\sin \theta \\
\sin \theta & \cos \theta
\end{array}\right)\left(\begin{array}{l}
x(t) \\
y(t)
\end{array}\right), \quad t \in[0, T],
$$

and let $A_{\theta}: C_{a, b}^{2}[0, T] \rightarrow C_{a, b}^{2}[0, T]$ be the transform defined by $A_{\theta}(x, y)=\left(X^{\prime}, Y^{\prime}\right)$ where

$$
\left(\begin{array}{l}
X^{\prime}(t) \\
Y^{\prime}(t)
\end{array}\right)=\left(\begin{array}{l}
x(t) \\
y(t)
\end{array}\right)-\mathbb{E}\left[\left(\begin{array}{l}
x(t) \\
y(t)
\end{array}\right)\right]+\mathbb{E}\left[R_{\theta}(x, y)(t)\right], \quad t \in[0, T]
$$

where $\mathbb{E}$ is the expectation, which implies that

$$
\left\{\begin{array}{l}
X^{\prime}(t)=(x(t)-a(t))+(\cos \theta-\sin \theta) a(t) \\
Y^{\prime}(t)=(y(t)-a(t))+(\cos \theta+\sin \theta) a(t), \quad t \in[0, T] .
\end{array}\right.
$$

Note that if $a(t) \equiv 0$ on $[0, T]$, then the transform $A_{\theta}$ is the identity transform on $C_{a, b}^{2}[0, T]$.

The following lemma plays a key role to obtain our main results and formulas of this paper.

Lemma 3.1. For any Borel set $B$ in $C_{a, b}^{2}[0, T]$,

$$
(\mu \times \mu) \circ R_{\theta}^{-1}(B)=(\mu \times \mu) \circ A_{\theta}^{-1}(B) .
$$

Proof. Let $\mathcal{B}\left(C_{a, b}[0, T]\right)$ and $\mathcal{B}\left(C_{a, b}^{2}[0, T]\right)$ be the Borel $\sigma$-algebras on $C_{a, b}[0, T]$ and $C_{a, b}^{2}[0, T]$, respectively. Since $C_{a, b}[0, T]$ is a separable metric space, we see that $\mathcal{B}\left(C_{a, b}^{2}[0, T]\right)=\mathcal{B}\left(C_{a, b}[0, T]\right) \times \mathcal{B}\left(C_{a, b}[0, T]\right)$ and that $\mathcal{B}\left(C_{a, b}^{2}[0, T]\right)$ is coincided with the $\sigma$-algebra generated by the collection of all cylinder sets on $C_{a, b}^{2}[0, T]$. Thus to prove our assertion, it suffices to show that

$$
(\mu \times \mu) \circ R_{\theta}^{-1}(I \times J)=(\mu \times \mu) \circ A_{\theta}^{-1}(I \times J),
$$

where $I$ and $J$ are cylinder sets on $C_{a, b}[0, T]$. For $0=t_{0}<t_{1}<\cdots<t_{n} \leq T$, let

$$
\begin{aligned}
I \times J=\{(X, Y) & \in C_{a, b}^{2}[0, T] \mid \\
& \left.\alpha_{j}<X\left(t_{j}\right) \leq \beta_{j}, \xi_{j}<Y\left(t_{j}\right) \leq \eta_{j}, j=1, \cdots, n\right\} .
\end{aligned}
$$


Then

$$
\begin{aligned}
R_{\theta}^{-1}(I \times J)=\{ & (x, y) \in C_{a, b}^{2}[0, T] \mid \alpha_{j}<x\left(t_{j}\right) \cos \theta-y\left(t_{j}\right) \sin \theta \leq \beta_{j}, \\
& \left.\xi_{j}<x\left(t_{j}\right) \sin \theta+y\left(t_{j}\right) \cos \theta \leq \eta_{j}, j=1, \cdots, n\right\}
\end{aligned}
$$

and

$$
\begin{gathered}
A_{\theta}^{-1}(I \times J)=\left\{(x, y) \in C_{a, b}^{2}[0, T] \mid \alpha_{j}<x\left(t_{j}\right)-a\left(t_{j}\right)+(\cos \theta-\sin \theta) a\left(t_{j}\right) \leq \beta_{j},\right. \\
\left.\xi_{j}<y\left(t_{j}\right)-a\left(t_{j}\right)+(\cos \theta+\sin \theta) a\left(t_{j}\right) \leq \eta_{j}, j=1, \cdots, n\right\} .
\end{gathered}
$$

Using equations (3.1), (3.2) and (3.3) it follows that

$$
\begin{aligned}
& (\mu \times \mu) \circ R_{\theta}^{-1}(I \times J) \\
& =\int_{C_{a, b}^{2}[0, T]} \chi_{R_{\theta}^{-1}(I \times J)}(x, y) d(\mu \times \mu)(x, y) \\
& =\int_{C_{a, b}^{2}[0, T]}\left[\prod_{j=1}^{n} \chi_{\left(\alpha_{j}, \beta_{j}\right]}\left(x\left(t_{j}\right) \cos \theta-y\left(t_{j}\right) \sin \theta\right)\right] \\
& \cdot\left[\prod_{j=1}^{n} \chi_{\left(\xi_{j}, \eta_{j}\right]}\left(x\left(t_{j}\right) \sin \theta+y\left(t_{j}\right) \cos \theta\right)\right] d(\mu \times \mu)(x, y) \\
& =\int_{\mathbb{R}^{n}} \int_{\mathbb{R}^{n}} \prod_{j=1}^{n} \chi_{\left(\alpha_{j}, \beta_{j}\right]}\left(u_{j} \cos \theta-v_{j} \sin \theta\right) \cdot \prod_{j=1}^{n} \chi_{\left(\xi_{j}, \eta_{j}\right]}\left(u_{j} \sin \theta+v_{j} \cos \theta\right) \\
&
\end{aligned}
$$

where $W_{n}(\vec{t}, \vec{u})$ is given by (2.1) above. Now, let $u_{j} \cos \theta-v_{j} \sin \theta=u_{j}^{\prime}$ and $u_{j} \sin \theta+v_{j} \cos \theta=v_{j}^{\prime}$ for each $j=1, \ldots, n$. Then the last expression above becomes

$$
\begin{gathered}
{\left[(2 \pi)^{n} \prod_{j=1}^{n}\left(b\left(t_{j}\right)-b\left(t_{j-1}\right)\right)\right]^{-1} \int_{\mathbb{R}^{n}} \int_{\mathbb{R}^{n}} \prod_{j=1}^{n} \chi_{\left(\alpha_{j}, \beta_{j}\right]}\left(u_{j}^{\prime}\right) \cdot \prod_{j=1}^{n} \chi_{\left(\xi_{j}, \eta_{j}\right]}\left(v_{j}^{\prime}\right)} \\
\cdot \exp \left\{-\frac{1}{2} \sum_{j=1}^{n} \frac{\left(u_{j}^{\prime}-u_{j-1}^{\prime}\right)^{2}+\left(v_{j}^{\prime}-v_{j-1}^{\prime}\right)^{2}+2\left(a\left(t_{j}\right)-a\left(t_{j-1}\right)\right)^{2}}{b\left(t_{j}\right)-b\left(t_{j-1}\right)}\right. \\
\left.-\frac{1}{2} \sum_{j=1}^{n} \frac{2\left(u_{j}^{\prime}-u_{j-1}^{\prime}\right)\left(a\left(t_{j}\right)-a\left(t_{j-1}\right)\right)(\sin \theta-\cos \theta)}{b\left(t_{j}\right)-b\left(t_{j-1}\right)}\right\} d \overrightarrow{u^{\prime}} d \overrightarrow{v^{\prime}} .
\end{gathered}
$$


By the way, let $u_{j}^{\prime}=u_{j}-a\left(t_{j}\right)+(\cos \theta-\sin \theta) a\left(t_{j}\right)$ and $v_{j}^{\prime}=v_{j}-a\left(t_{j}\right)+(\cos \theta+$ $\sin \theta) a\left(t_{j}\right)$ for each $j=1, \ldots, n$. Then the last expression above becomes

$$
\begin{aligned}
& \int_{\mathbb{R}^{n}} \int_{\mathbb{R}^{n}} \prod_{j=1}^{n} \chi_{\left(\alpha_{j}, \beta_{j}\right]}\left(u_{j}-a\left(t_{j}\right)+(\cos \theta-\sin \theta) a\left(t_{j}\right)\right) \\
& \cdot \prod_{j=1}^{n} \chi_{\left(\xi_{j}, \eta_{j}\right]}\left(v_{j}-a\left(t_{j}\right)+(\cos \theta+\sin \theta) a\left(t_{j}\right)\right) \\
& \cdot W_{n}(\vec{t}, \vec{u}) W_{n}(\vec{t}, \vec{v}) d \vec{u} d \vec{v} \\
& =\int_{C_{a, b}^{2}[0, T]}\left[\prod_{j=1}^{n} \chi_{\left(\alpha_{j}, \beta_{j}\right]}\left(x\left(t_{j}\right)-a\left(t_{j}\right)+(\cos \theta-\sin \theta) a\left(t_{j}\right)\right)\right] \\
& \cdot\left[\prod_{j=1}^{n} \chi_{\left(\xi_{j}, \eta_{j}\right]}\left(y\left(t_{j}\right)-a\left(t_{j}\right)+(\cos \theta+\sin \theta) a\left(t_{j}\right)\right)\right] d(\mu \times \mu)(x, y) \\
& =\int_{C_{a, b}^{2}[0, T]} \chi_{A_{\theta}^{-1}(I \times J)}(x, y) d(\mu \times \mu)(x, y) \\
& =(\mu \times \mu) \circ A_{\theta}^{-1}(I \times J),
\end{aligned}
$$

which completes the proof of the Lemma 3.1 as desired.

Remark 3.2. Note that $R_{\theta}^{-1}(N)$ is a Borel null set if and only if $A_{\theta}^{-1}(N)$ is a Borel null set. Hence, by the Carathéodory extension, we see that for any $\mu \times \mu$ measurable set $E,(\mu \times \mu) \circ R_{\theta}^{-1}(E)=(\mu \times \mu) \circ A_{\theta}^{-1}(E)$.

Theorem 3.3. Let $G$ be a complex-valued functional on $C_{a, b}^{2}[0, T]$. Then $G\left(R_{\theta}(x, y)\right)$ is $\mu \times \mu$-measurable if and only if $G\left(A_{\theta}(x, y)\right)$ is $\mu \times \mu$-measurable and

$$
\int_{C_{a, b}^{2}[0, T]} G\left(R_{\theta}(x, y)\right) d(\mu \times \mu)(x, y) \stackrel{*}{=} \int_{C_{a, b}^{2}[0, T]} G\left(A_{\theta}(x, y)\right) d(\mu \times \mu)(x, y)
$$

where by $\stackrel{*}{=}$ we means that if either side exists, both sides exist and equality holds. Proof. The proof of Theorem 3.3 is straightforward by Lemma 3.1.

Lemma 3.4. Let $F$ be a complex-valued Borel measurable functional on $C_{a, b}[0, T]$. Then

$$
\begin{aligned}
& \int_{C_{a, b}^{2}[0, T]} F(x \sin \theta+y \cos \theta) d(\mu \times \mu)(x, y) \\
& \stackrel{*}{=} \int_{C_{a, b}[0, T]} F(z+(\sin \theta+\cos \theta-1) a) d \mu(z)
\end{aligned}
$$

where $a \equiv a(t)$ is as in Remark 2.1.

Proof. Let $P_{2}: C_{a, b}^{2}[0, T] \rightarrow C_{a, b}[0, T]$ be the projection map given by $P_{2}(x, y)=y$. Then equation (3.5) follows from equation (3.4) with $G$ replaced with $F \circ P_{2}$.

The following theorem is the first main result in this paper, which is called a Fubini theorem on function spaces. 
Theorem 3.5. (Fubini theorem) Let $F$ be a complex-valued Borel measurable functional on $C_{a, b}[0, T]$ such that

$$
\int_{C_{a, b}^{2}[0, T]}|F(p x+q y)| d(\mu \times \mu)(x, y)<\infty
$$

for all non-zero real numbers $p$ and $q$. Then for all $p, q \in \mathbb{R}-\{0\}$,

$$
\begin{aligned}
& \int_{C_{a, b}^{2}[0, T]} F(p x+q y) d(\mu \times \mu)(x, y) \\
& =\int_{C_{a, b}[0, T]} F\left(\sqrt{p^{2}+q^{2}} z+\left(p+q-\sqrt{p^{2}+q^{2}}\right) a\right) d \mu(z),
\end{aligned}
$$

where $a(t)$ is as in Remark 2.1.

Proof. For given $p$ and $q$, letting $\sin \theta=\frac{p}{\sqrt{p^{2}+q^{2}}}, \cos \theta=\frac{q}{\sqrt{p^{2}+q^{2}}}$ and $H(x)=$ $F\left(\sqrt{p^{2}+q^{2}} x\right)$ and using equation (3.5) with $F$ replaced with $H$, we obtain that

$$
\begin{aligned}
& \int_{C_{a, b}^{2}[0, T]} F(p x+q y) d(\mu \times \mu)(x, y) \\
& =\int_{C_{a, b}^{2}[0, T]} F\left(\sqrt{p^{2}+q^{2}}\left(\frac{p}{\sqrt{p^{2}+q^{2}}} x+\frac{q}{\sqrt{p^{2}+q^{2}}} y\right)\right) d(\mu \times \mu)(x, y) \\
& =\int_{C_{a, b}[0, T]} H(x \sin \theta+y \cos \theta) d \mu(z) \\
& =\int_{C_{a, b}[0, T]} H(z+(\sin \theta+\cos \theta-1) a) d \mu(z) \\
& =\int_{C_{a, b}[0, T]} F\left(\sqrt{p^{2}+q^{2}} z+\left(p+q-\sqrt{p^{2}+q^{2}}\right) a\right) d \mu(z),
\end{aligned}
$$

which completes the proof of the Theorem 3.5 as desired.

In the following example, we illustrate some usefulness of our Fubini theorem. First of all, we will use the following well-known Wiener integration formula several times in our calculations.

Theorem 3.6. Let $f: \mathbb{R} \rightarrow \mathbb{C}$ be a Lebesgue measurable function and let $v$ be an element of $L_{a, b}^{2}[0, T]$. If $F(x)=f(\langle v, x\rangle)$, then $F$ is $\mu$-measurable and

$$
\int_{C_{a, b}[0, T]} F(x) d \mu(x) \stackrel{*}{=}\left(\frac{1}{2 \pi\left(v^{2}, b^{\prime}\right)}\right)^{\frac{1}{2}} \int_{\mathbb{R}} f(u) \exp \left\{-\frac{\left(u-\left(v, a^{\prime}\right)\right)^{2}}{2\left(v^{2}, b^{\prime}\right)}\right\} d u .
$$

Example 3.7. Let $f(u)=u^{2}$ and let $F(x)=f(\langle v, x\rangle)=\langle v, x\rangle^{2}$. Then we easily see that for all $p, q \in \mathbb{R}-\{0\}$,

$$
\int_{C_{a, b}^{2}[0, T]}|F(p x+q y)| d(\mu \times \mu)(x, y)<\infty .
$$


We note that for all $p \in \mathbb{R}-\{0\}$, using equation (3.7),

$$
\int_{C_{a, b}[0, T]} F(p x) d \mu(x)=p^{2}\left(v^{2}, b^{\prime}\right)+p\left(v, a^{\prime}\right)^{2} .
$$

This means that $F$ satisfies the hypotheses of Theorem 3.5, and hence using equation (3.6), it follows that for all $p, q \in \mathbb{R}-\{0\}$,

$$
\begin{aligned}
& \int_{C_{a, b}^{2}[0, T]} F(p x+q y) d(\mu \times \mu)(x, y) \\
& =\int_{C_{a, b}[0, T]} F\left(\sqrt{p^{2}+q^{2}} z+\left(p+q-\sqrt{p^{2}+q^{2}}\right) a\right) d \mu(z) \\
& =\left(p^{2}+q^{2}\right)\left(v^{2}, b^{\prime}\right)+(p+q)^{2}\left(v, a^{\prime}\right)^{2} .
\end{aligned}
$$

Next, we give an example more complicated than Example 3.7 above.

Example 3.8. Let $f(u)=e^{-u^{2}}$ and let $F(x)=f(\langle v, x\rangle)=\exp \left\{-\langle v, x\rangle^{2}\right\}$. Then we easily see that for all $p, q \in \mathbb{R}-\{0\}$,

$$
\int_{C_{a, b}^{2}[0, T]}|F(p x+q y)| d(\mu \times \mu)(x, y)<\infty .
$$

We note that for all $p \in \mathbb{R}-\{0\}$, using equation (3.7),

$$
\int_{C_{a, b}[0, T]} F(p x) d \mu(x)=\frac{1}{\sqrt{2 p^{2}\left(v^{2}, b^{\prime}\right)+1}} \exp \left\{-\frac{p^{2}\left(v, a^{\prime}\right)^{2}}{2 p^{2}\left(v^{2}, b^{\prime}\right)+1}\right\} .
$$

This means that $F$ satisfies the hypotheses of Theorem 3.5, and hence using equation (3.6), it follows that for all $p, q \in \mathbb{R}-\{0\}$,

$$
\begin{aligned}
& \int_{C_{a, b}^{2}[0, T]} F(p x+q y) d(\mu \times \mu)(x, y) \\
& =\int_{C_{a, b}[0, T]} F\left(\sqrt{p^{2}+q^{2}} z+\left(p+q-\sqrt{p^{2}+q^{2}}\right) a\right) d \mu(z) \\
& =\frac{1}{\sqrt{2\left(p^{2}+q^{2}\right)\left(v^{2}, b^{\prime}\right)+1}} \exp \left\{-\frac{(p+q)^{2}\left(v, a^{\prime}\right)^{2}}{2\left(p^{2}+q^{2}\right)\left(v^{2}, b^{\prime}\right)+1}\right\} .
\end{aligned}
$$

\section{Applications}

In this section we use the Fubini theorem and the translation theorem to obtain interesting relationships involving a Cameron-Storvick type theorem, see Theorems 4.2 and 4.6 below.

The following lemma was established in [9, p.379]. 
Lemma 4.1. (Translation theorem) Let $x_{0}(t)=\int_{0}^{t} u(s) d b(s)$ for some $u \in$ $L_{a, b}^{2}[0, T]$ and let $F$ be a $\mu$-integrable functional on $C_{a, b}[0, T]$. Then

$$
\begin{aligned}
\int_{C_{a, b}[0, T]} & F\left(x+x_{0}\right) d \mu(x) \\
& =\exp \left\{-\frac{1}{2}\left(u^{2}, b^{\prime}\right)-\left(u, a^{\prime}\right)\right\} \int_{C_{a, b}[0, T]} F(x) \exp \{\langle u, x\rangle\} d \mu(x) .
\end{aligned}
$$

Now we establish an interesting relationship between the translation theorem and the our Fubini theorem.

Theorem 4.2. (Application 1) Let the function $a \equiv a(t)$ and $w$ be as in Remark 2.1 and let $F$ be as in Theorem 3.5 above. Then for all $p, q \in \mathbb{R}-\{0\}$,

$$
\begin{aligned}
\int_{C_{a, b}^{2}[0, T]} F(p x+q y) d(\mu \times \mu)(x, y) & =\exp \left\{-\frac{p q}{p^{2}+q^{2}}\left(w, a^{\prime}\right)\right\} \\
& \cdot \int_{C_{a, b}[0, T]} F\left(\sqrt{p^{2}+q^{2}} z\right) \exp \left\{\frac{p+q-\sqrt{p^{2}+q^{2}}}{\sqrt{p^{2}+q^{2}}}\langle w, z\rangle\right\} d \mu(z) .
\end{aligned}
$$

Proof. The proof is straightforward by applying the Fubini theorem (Theorem 3.5) and the translation theorem (Lemma 4.1).

The following corollary immediately follows from Theorem 4.2.

Corollary 4.3. (1) Let $a, w$ and $F$ be as in Theorem 4.2 above. Then for all non-zero real numbers $p$ and $q$ with $p^{2}+q^{2}=1$,

$$
\begin{aligned}
& \int_{C_{a, b}^{2}[0, T]} F(p x+q y) d(\mu \times \mu)(x, y) \\
& =\exp \left\{-p q\left(w, a^{\prime}\right)\right\} \int_{C_{a, b}[0, T]} F(z) \exp \{(p+q-1)\langle w, z\rangle\} d \mu(z) .
\end{aligned}
$$

(2) When $a(t) \equiv 0$ on $[0, T]$ and hence $w(t) \equiv 0$ on $[0, T]$, it follows that for all non-zero real numbers $p$ and $q$,

$$
\int_{C_{a, b}^{2}[0, T]} F(p x+q y) d(\mu \times \mu)(x, y)=\int_{C_{a, b}[0, T]} F\left(\sqrt{p^{2}+q^{2}} z\right) d \mu(z) .
$$

We are ready to state the definition of the first variation of a functional $F$ on a function space.

Definition 4.4. Let $F$ be a complex-valued functional on $C_{a, b}[0, T]$. Then

$$
\delta F(x \mid h)=\left.\frac{\partial}{\partial k} F(x+k h)\right|_{k=0}, \quad x, h \in C_{a, b}[0, T],
$$

(if it exists) is called the first variation of $F$ in the direction of $h$. 
The following lemma is very useful to find a Cameron-Storvick type theorem on a function space. This is a straightforward extension of the Application 1, namely Theorem 4.2 above.

Lemma 4.5. Let $F, w$ and $a$ be as in Theorem 4.2 above. Then for $u \in C_{a, b}[0, T]$ and all non-zero real numbers $p$ and $q$,

$$
\begin{aligned}
& \int_{C_{a, b}^{2}[0, T]} F(p x+q y+u) d(\mu \times \mu)(x, y) \\
& =\exp \left\{-\frac{p q}{p^{2}+q^{2}}\left(w, a^{\prime}\right)\right\} \\
& \cdot \int_{C_{a, b}[0, T]} F\left(\sqrt{p^{2}+q^{2}} z+u\right) \exp \left\{\frac{p+q-\sqrt{p^{2}+q^{2}}}{\sqrt{p^{2}+q^{2}}}\langle w, z\rangle\right\} d \mu(z) .
\end{aligned}
$$

The following theorem is the last theorem in this paper which is called a Cameron-Storvick type theorem on function spaces.

Theorem 4.6. (Application 2 : Cameron-Storvick type theorem) Let F, w and $a$ be as in Theorem 4.2 above, and let $h(t)=\int_{0}^{t} v(s) d b(s)$ for some $v \in L_{a, b}^{2}[0, T]$. Assume that

$$
\int_{C_{a, b}^{2}[0, T]}|\delta F(p x+q y \mid h)| d(\mu \times \mu)(x, y)<\infty
$$

for all non-zero real numbers $p$ and $q$. Then for all non-zero real numbers $p$ and $q$,

$$
\begin{aligned}
& \int_{C_{a, b}^{2}[0, T]} \delta F(p x+q y \mid h) d(\mu \times \mu)(x, y) \\
& =\frac{1}{\sqrt{p^{2}+q^{2}}} \exp \left\{-\frac{p q}{p^{2}+q^{2}}\left(w, a^{\prime}\right)\right\} \\
& \quad \cdot \int_{C_{a, b}[0, T]}\langle v, z\rangle F\left(\sqrt{p^{2}+q^{2}} z\right) \exp \left\{\frac{p+q-\sqrt{p^{2}+q^{2}}}{\sqrt{p^{2}+q^{2}}}\langle w, z\rangle\right\} d \mu(z) \\
& -\frac{p+q}{p^{2}+q^{2}}\left(v, a^{\prime}\right) \exp \left\{-\frac{p q}{p^{2}+q^{2}}\left(w, a^{\prime}\right)\right\} \\
& \cdot \int_{C_{a, b}[0, T]} F\left(\sqrt{p^{2}+q^{2}} z\right) \exp \left\{\frac{p+q-\sqrt{p^{2}+q^{2}}}{\sqrt{p^{2}+q^{2}}}\langle w, z\rangle\right\} d \mu(z) .
\end{aligned}
$$


Proof. First, by applying the dominated convergence theorem and using equation (4.1), we obtain that for all non-zero real numbers $p$ and $q$,

$$
\begin{aligned}
& \int_{C_{a, b}^{2}[0, T]} \delta F(p x+q y \mid h) d(\mu \times \mu)(x, y) \\
& =\left.\int_{C_{a, b}^{2}[0, T]} \frac{\partial}{\partial k} F(p x+q y+k h)\right|_{k=0} d(\mu \times \mu)(x, y) \\
& =\frac{\partial}{\partial k}\left[\exp \left\{-\frac{p q}{p^{2}+q^{2}}\left(w, a^{\prime}\right)\right\} \int_{C_{a, b}[0, T]} F\left(\sqrt{p^{2}+q^{2}} z+k h\right)\right. \\
& \left.\cdot \exp \left\{\frac{p+q-\sqrt{p^{2}+q^{2}}}{\sqrt{p^{2}+q^{2}}}\langle w, z\rangle\right\} d \mu(z)\right]\left.\right|_{k=0} .
\end{aligned}
$$

The remainder of the proof is straightforward by applying the translation theorem (Lemma 4.1) and taking the partial derivative in variable $k$.

The following corollary immediately follows from Theorem 4.6.

Corollary 4.7. Let $F, w, h$ and $a$ be as in Theorem 4.6 above. Then for all non-zero real numbers $p$ and $q$ with $p^{2}+q^{2}=1$,

$$
\begin{aligned}
& \int_{C_{a, b}^{2}[0, T]} \delta F(p x+q y \mid h) d(\mu \times \mu)(x, y) \\
& =\exp \left\{-p q\left(w, a^{\prime}\right)\right\} \int_{C_{a, b}[0, T]}\langle v, z\rangle F(z) \exp \{(p+q-1)\langle w, z\rangle\} d \mu(z) \\
& -(p+q)\left(v, a^{\prime}\right) \exp \left\{-p q\left(w, a^{\prime}\right)\right\} \\
& \cdot \int_{C_{a, b}[0, T]} F(z) \exp \{(p+q-1)\langle w, z\rangle\} d \mu(z) .
\end{aligned}
$$

Remark 4.8. As mentioned in Section 3, we can apply our applications in Theorem 4.2 and 4.6 above to various classes of functionals. Also, as discussed in $[16,17]$, we can apply our result obtained in Sections 3 and 4 to study various topics related with the generalized analytic Feynman integral and the generalized analytic Fourier-Feynman transform for functionals on $C_{a, b}[0, T]$.

\section{HiSTORICAL REMARKS}

In this section we present some remarks for our main theorems obtained in Sections 3 and 4 above.

\section{For Fubini theorem.}

In the setting of one parameter Wiener space $C_{0}[0, T]$ (i.e., in the case where $a(t) \equiv 0$ and $b(t)=t$ on $[0, T]$ in our research), Bearman [1] studied a significant rotation property for double Wiener integral. Cameron and Storvick developed the Bearman's result to study an operator valued Yeh-Wiener integral and a Wiener integral equation [2, Lemma 2]. The result, as a corollary of Theorem 3.5 , is summarized as follows. 
For an appropriate $F: C_{0}[0, T] \rightarrow \mathbb{C}$ and non-zero real numbers $p$ and $q$,

$$
\int_{C_{0}^{2}[0, T]} F(p w+q z) d(m \times m)(w, z)=\int_{C_{0}[0, T]} F\left(\sqrt{p^{2}+q^{2}} x\right) d m(x)
$$

where $m$ is the Wiener measure. This rotation theorem was improved in [18] to study the analytic Feynman integral and the analytic Fourier-Feynman transform theories. For a detailed study of the theories, see [18, Section 5]. Also, see $[4,5,9,11,13,14,15,16,17,20]$ for related work.

\section{For Cameron-Storvick type theorem.}

In the setting of one parameter Wiener space $C_{0}[0, T]$, Cameron and Storvick (See $\left[20\right.$, p.278]) established that for appropriate $F: C_{0}[0, T] \rightarrow \mathbb{C}$,

$$
\int_{C_{0}[0, T]} \delta F(z \mid h) d m(z)=\int_{C_{0}[0, T]}\langle v, z\rangle F(z) d m(z)
$$

where $h$ is given by $h(t)=\int_{0}^{t} v(s) d s$ for $v \in L_{2}[0, T]$ and $m$ is the Wiener measure. Whereas in [9], Chang and Skoug showed that

$$
\int_{C_{a, b}[0, T]} \delta F(z \mid h) d \mu(z)=\int_{C_{a, b}[0, T]}\langle v, z\rangle F(z) d \mu(z)-\left(v, a^{\prime}\right) \int_{C_{a, b}[0, T]} F(z) d \mu(z),
$$

where $h$ is given by $h(t)=\int_{0}^{t} v(s) d b(s)$ for $v \in L_{a, b}^{2}[0, T]$. One can see that these results are immediate corollaries of Theorem 4.6 above.

Acknowledgement. The authors would like to express their gratitude to the referees for their valuable comments and suggestions which have improved the original manuscript. This research was supported by Basic Science Research Program through the National Research Foundation of Korea(NRF) funded by the Ministry of Education, Science and Technology(2012R1A1A1004774 : 2011$0014552)$

\section{REFERENCES}

1. J.E. Bearman, Rotations in the product of two Wiener spaces, Proc. Amer. Math. Soc. 3 (1952), no. 1, 129-137.

2. R.H. Cameron and D.A. Storvick, An operator valued Yeh-Wiener integral, and a Wiener integral equation, Indiana Univ. Math. J. 25 (1976), no. 3, 235-258.

3. R.H. Cameron and D.A. Storvick, Feynman integral of variation of functionals, in: Gaussian Random Fields, World Scientific, 144-157, Singapore, 1991.

4. S.J. Chang, J.G. Choi and H.S. Chung, Generalized analytic Feynman integral via function space integral of bounded cylinder functionals, Bull. Korean Math. Soc. 48 (2011), no. 3, $475-489$.

5. S.J. Chang, J.G. Choi and D. Skoug, Integration by parts formulas involving generalized Fourier-Feynman transforms on function space, Trans. Amer. Math. Soc. 355 (2003), no. 7, 2925-2948.

6. S.J. Chang and D.M. Chung, Conditional function space integrals with applications, Rocky Mountain J. Math. 26 (1996), no. 1, 37-62.

7. S.J. Chang and H.S. Chung, Generalized Fourier-Wiener function space transforms, J. Korean Math. Soc. 46 (2009), no. 2, 327-345. 
8. S.J. Chang, H.S. Chung and D. Skoug, Integral transforms of functionals in $L^{2}\left(C_{a, b}[0, T]\right)$, J. Fourier Anal. Appl. 15 (2009), no. 4, 441-462.

9. S.J. Chang and D. Skoug, Generalized Fourier-Feynman transforms and a first variation on function space, Integral Transforms Spec. Funct. 14 (2003), no. 5, 375-393.

10. H.S. Chung and S.J. Chang, Some applications of the spectral theory for the integral transform involving the spectral representation, J. Funct. spaces Appl. 2010, Art. ID 573602, 17 pp.

11. H.S. Chung and V.K. Tuan, A sequential analytic Feynman integral of functionals in $L_{2}\left(C_{0}[0, T]\right)$, Integral Transforms Spec. Funct. 23 (2012), no. 7, 495-502.

12. H.S. Chung and V.K. Tuan, Fourier-type functionals on Wiener space, Bull. Korean Math. Soc. 49 (2012), no. 3, 609-619.

13. T. Huffman, C. Park and D. Skoug, Analytic Fourier-Feynman transforms and convolution, Trans. Amer. Math. Soc. 347 (1995), no. 2, 661-673.

14. T. Huffman, C. Park and D. Skoug, Convolutions and Fourier-Feynman transforms of functionals involving multiple integrals, Michigan Math. J. 43 (1996), no. 2, 247-261.

15. T. Huffman, C. Park and D. Skoug, Convolutions and Fourier-Feynman transforms, Rocky Mountain J. Math. 27 (1997), no. 3, 827-841.

16. T. Huffman, C. Park and D. Skoug, A Fubini theorem for analytic Feynman integrals with applications, J. Korean Math. Soc. 38 (2001), no. 2, 409-420.

17. T. Huffman, C. Park and D. Skoug, Integration formulas involving Fourier-Feynman transforms via a Fubini theorem, J. Korean Math. Soc. 38 (2001), no. 2, 421-435.

18. G.W. Johnson and D.L. Skoug, Scale-invariant measurability in Wiener space, Pacific J. Math. 83(1979), no. 1, 157-176.

19. E. Nelson, Dynamical Theories of Brownian Motion (2nd edition), Math. Notes, Princeton University Press, Princeton, 1967.

20. C. Park, D. Skoug and D.A. Storvick, Fourier-Feynman transforms and the first variation, Rend. Circ. Mat. Palermo (2) 47 (1998), no. 2, 277-292.

21. J. Yeh, Stochastic Processes and the Wiener Integral, Marcel Dekker, Inc., New York, 1973.

22. J. Yeh, Singularity of Gaussian measures on function spaces induced by Brownian motion processes with non-stationary increments, Illinois J. Math. 15 (1971), no. 1, 37-46.

Department of Mathematics, Dankook University, Cheonan 330-714, Korea, E-mail address: hschung@dankook.ac.kr

E-mail address: jgchoi@dankook.ac.kr

E-mail address: sejchang@dankook.ac.kr 